\title{
Soccer Passing Accuracy Differentiates Between High and Low Digit Ratio (2D:4D) Soccer Players
}

\author{
Muhammad Shahidul Islam ${ }^{1,2, ~ *, ~ B r a j a n a t h ~ K u n d u ~}{ }^{1}$ \\ ${ }^{1}$ Department of Physical Education and Sport Science, Visva-Bharati University, Santiniketan, India \\ ${ }^{2}$ Office of Physical Education, Shahjalal University of Science and Technology, Sylhet, Bangladesh
}

Email address:

jewel.soccer@yahoo.com (M. S. Islam), brajanathvb@gmail.com (B. Kundu)

${ }^{*}$ Corresponding author

\section{To cite this article:}

Muhammad Shahidul Islam, Brajanath Kundu. Soccer Passing Accuracy Differentiates Between High and Low Digit Ratio (2D:4D) Soccer Players. American Journal of Sports Science. Vol. 8, No. 3, 2020, pp. 49-55. doi: 10.11648/j.ajss.20200803.11

Received: June 20, 2020; Accepted: July 7, 2020; Published: July 17, 2020

\begin{abstract}
The purpose of the present study was to know soccer passing accuracy performances between the national youth level soccer players possessing high digit ratio and low digit ratio (2D:4D). The study was carried out in 108 male soccer players of Bangladesh aged between 16 to 19 years for measured the digit ratio. To find out the high and low digit ratio groups of participant quartile deviation has been used. Participants belonged to 0.973 and above $(\mathrm{N}=28)$ were categorized into high digit ratio group and 0.942 and below $(\mathrm{N}=28)$ were categorized into low digit ratio group. Soccer passing accuracy performance was assessed $(\mathrm{N}=56)$ by using Loughborough Soccer Passing Test (LSPT). Digit ratio (2D:4D) was measured by digital vernier caliper, Mitutoyo Corporation, Japan measuring to $0.01 \mathrm{~mm}$. The length of the index finger (2D) and ring finger (4D) measured in millimeters from the second metacarpophalangeal crease of the finger to the fingertip. The mean digit ratio was calculated as dividing the length of the $2 \mathrm{D}$ by the length of the $4 \mathrm{D}$ for the left and right hand one by one and then computes the mean of these two ratios. To find out the significance of differences of soccer passing accuracy between the high digit ratio and the low digit ratio groups independent t-test was used. It was observed that the mean score in soccer passing accuracy performance, i.e., LSPT Total performance (TP) of the lower digit ratio, group was a significantly better performer than that of the high digit ratio group. A higher level of fetal testosterone, characterized by lower values of the digit finger ratio (2D:4D), may be associated with a higher performance of soccer passing accuracy in multifaceted aspects including passing, dribbling, controlling, turning, decision-making, and cognition.
\end{abstract}

Keywords: Digit Ratio, Fetal Testosterone, Fetal Estrogen, Hormone, Passing Accuracy, Soccer

\section{Introduction}

The index finger length to ring finger length ratio (2D:4D) is considered a possible biomarker of prenatal androgen exposure [1]. Prenatal androgen exposure, with testosterone hormone being the most important androgen, plays an important role in mammalian brain sexual differentiation which has a lasting effect on Games, Sports, Physical and Competitive Behavior [2-8]. Digit ratio is claimed to be affected by the fetal exposure to the prenatal androgen. Analysis of amniocentesis samples shows that the digit ratio is negatively associated with prenatal testosterone hormone, but positively linked to exposure to estrogen hormone [9]. It is expected that a fetus with more testosterone exposure can have a lower digit ratio (masculine ratio). Male fetuses usually have a higher exposure to testosterone and therefore males always have lower digit ratios than females [10]. So, the ratio $2 \mathrm{D}: 4 \mathrm{D}$ is a prospective marker for prenatal testosterone and may describe differences in sports performance $[5,11]$. Most correlational early studies highlighted the negative relationships between lower digit ratio and performance in sports. Low second to fourth digit ratio (2D:4D) may be an indicator for prospective athletes, particularly soccer players $[6,26]$.

Soccer is an art and includes thousands of decisions deciding the result of the match [12]. Elite soccer players require high levels of cognitive, perceptive, and motor skills in a rapidly changing environment, [13-17] as the game is very intelligible [18]. Thus the cognitive ability plays an 
important role in performing a soccer skill [19] because soccer moments are mostly unpredicted and it hides on the brain of the player.

Quick short passing accuracy is considered a relevant skill for keeping ball possession [20]. Loughborough Soccer Passing Test (LSPT) assesses the multifaceted aspects of soccer skills including passing, dribbling, turning, decision making, and cognitive function [21]. This test included dynamic elements so that players have to decide by the caller's signal [22]. Hence, the test requires Visiospatial skills to better perform soccer. According to Chai \& Jacobs,[23] "The mental rotation task has become the standard spatial visualization task for studying the effects of digit ratio on spatial cognition" (p. 6). Spatial visualization with Visio-spatial perceptual pace and mental rotation is important for the interpretation of spatial mental representations. Peters et al. [24] highlighted the relationship between digit ratio and mental rotation on male and female and showed a strong negative association between the left and right digit ratio and mental rotation task ratings, which meant that individuals with low digit ratios (relatively longer ring finger than index finger) scored better than individuals with high digit ratios. Similarly, performing soccer skill is depending on creativity where players must be able to quickly and accurately perceive relevant information that promotes decision making [19].

Interestingly, earlier studies argued that the notable relationship between the masculine digit ratio (lower digit ratio) and the visual-spatial capacity [2, 25]. High prenatal testosterone exposure is likely to predict soccer performance as well as cognitive function [2]. Professor John Manning emphasizes the importance of Visio-spatial skills for soccer players. Manning, [26] stated "striking a moving opponent or ball requires fine judgment of distance. Determining the exact point of impact demands an accurate perception of the surface of the target as it moves through space" (p. 128). In addition, Researchers Acar \& Tutkun [3] analyzed the relationship between digit ratio of national and amateur soccer players and found in national soccer players, averages of both right and left-hand 2D:4D was found to be lower than the amateur soccer players. Altogether, the low digit ratio (2D:4D) may be associated with high soccer skill performance. Furthermore, in our knowledge, there is a gap of research concerning the comparative analysis of soccer passing accuracy skill between the high and low digit ratio (2D:4D) players. Consequently, this study aimed to compare the selected soccer passing accuracy performance among national youth soccer players with a high-digit ratio group, i.e., 0.973 and above $(\mathrm{N}=28)$ and a low-digit ratio group, i.e., 0.942 and below $(\mathrm{N}=28)$.

\section{Objective of the Study}

To compare the selected soccer passing accuracy performances (assessed by Loughborough Soccer Passing Test (LSPT)) between the national youth level soccer players possessing high digit ratio and low digit ratio (2D:4D).

\section{Methods}

\subsection{Selection of Participants}

The participants for this study were purposively selected from the national youth level soccer players in Bangladesh. The players were selected from various recognized soccer academies and youth clubs in Bangladesh. A total of 108 male soccer players, aged ranged between 16-19 years were selected for this study. All the players were field players from different playing positions and Goalkeepers were excluded from this study. All participants had normal vision and were right and left-handed both. Participants were informed verbally and in writing of the nature and requirements of the study. Written informed consent was obtained from all participants after the completion of the health questionnaire. The "Board of Studies," Department of Physical Education and Sport Science, Visva-Bharati University, carried out this study and forwarded it to the Institutional Research Board (University). Finally, the study was approved by the Research Board of the University.

\subsection{Hypothesis $\mathrm{H}_{1}$}

There would be significant differences in soccer passing accuracy skills between the high digit ratio and low digit ratio soccer players.

\section{Mean Digit Ratio (2D:4D)}

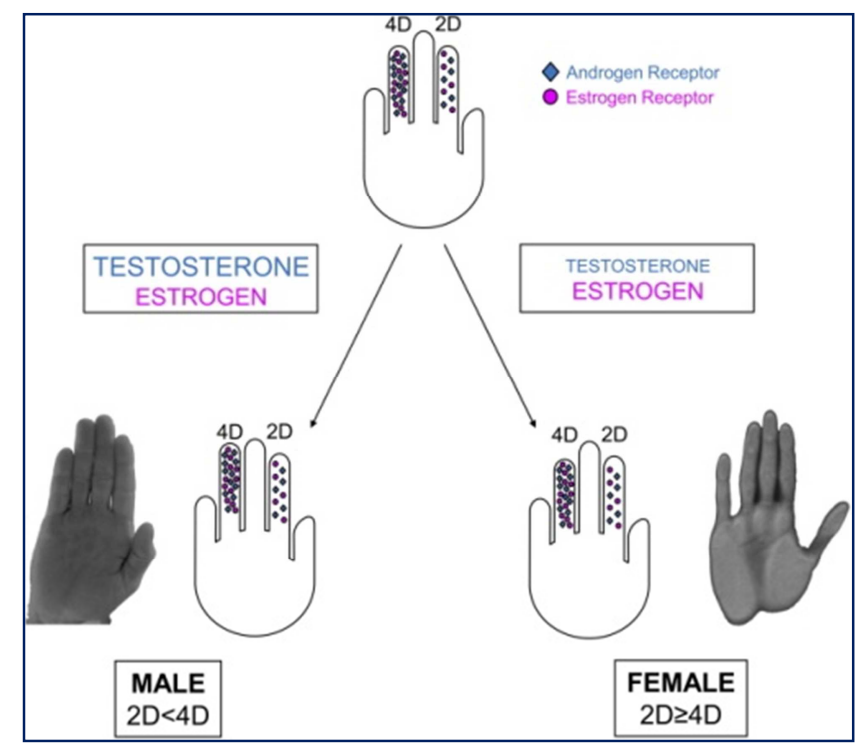

Figure 1. Ring finger denotes the testosterone hormone and index finger denotes the estrogen hormone prenatally [27].

Fingers (index and ring) of all the participants of the study were measured with the help of a digital vernier caliper, Model No. CD-6" CS Mitutoyo Corporation, Japan measuring to 0.01 $\mathrm{mm}$. The length of the index finger (2D) and ring finger (4D) measured in millimeters from the basal (lowest) crease of the finger to the fingertip along the bisecting medial line. All the measurements were taken twice. Digit ratio was calculated by dividing the length of the second digit (2D) by the length of 
the fourth digit (4D) for the left and right hand separately and then calculating the mean of these two ratios. Ring finger denotes the hormone of the prenatal testosterone and Index finger denotes the hormone of the estrogen in the womb of the mother (Figure 1).

Categorization into High and Low Digit Ratio (2D:4D)

Digit Ratio was categorized into high Digit Ratio and low Digit Ratio of the participants $(\mathrm{N}=108)$ using quartile deviation. It was observed that the Digit Ratio of the participants belonged to 0.973 and above ( $75 \%$ and above) were categorized into high Digit Ratio group and 0.942 and below (25\% and below) were categorized into low Digit Ratio group. Analyzing this quartile deviation calculation, it was observed that among the participants, 28 participants were high digit ratio group and 28 were under low digit ratio group.

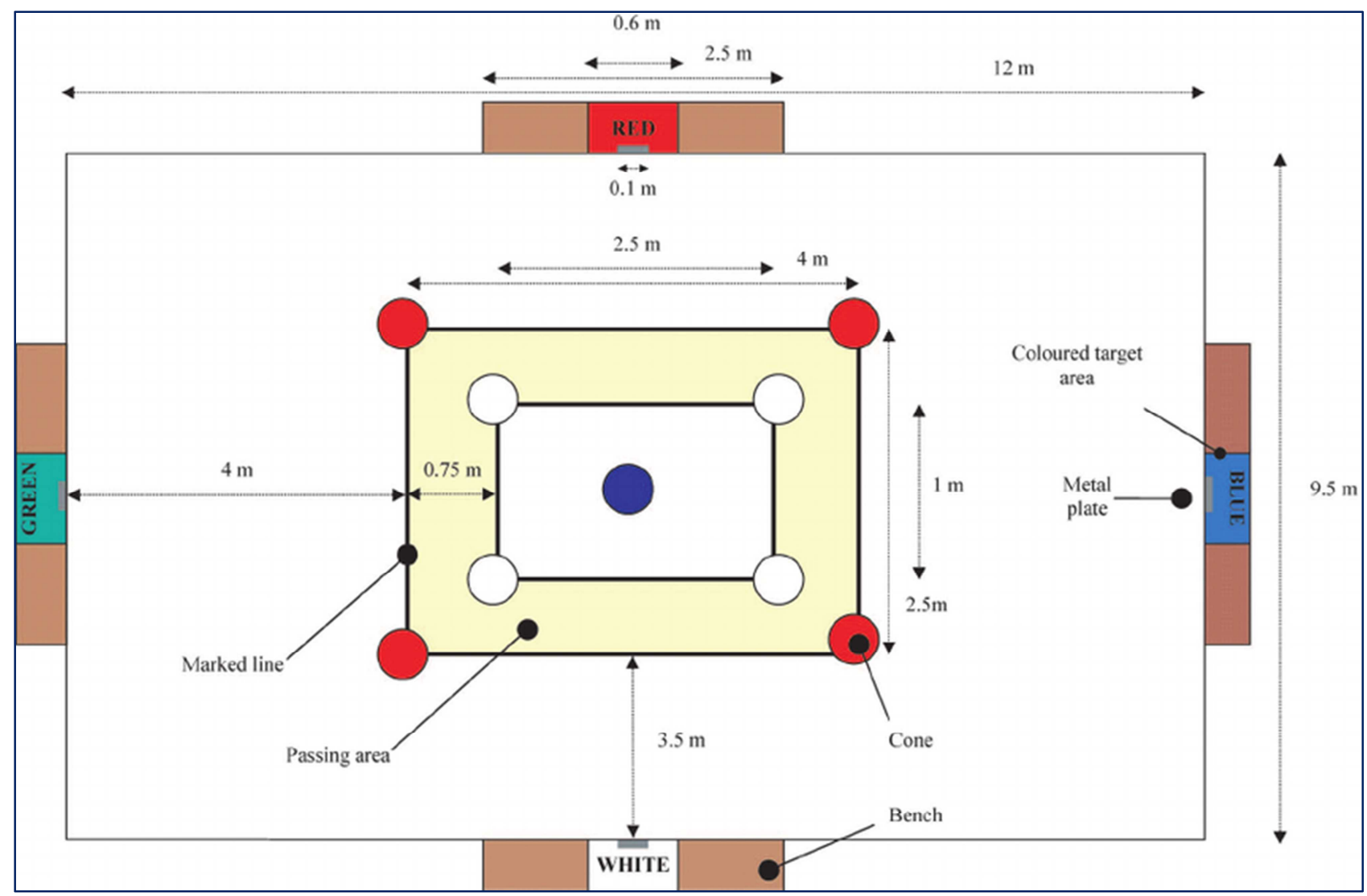

Figure 2. Schematic illustration of LSPT [22].

\section{Loughborough Soccer Passing Test (LSPT)}

Loughborough Soccer Passing Test (LSPT), a reliable and valid examination of passing ability in soccer players [22]. In this investigation, LSPT examined only selected group players with high $(\mathrm{N}=28)$ and low $(\mathrm{N}=28)$ digit ratios. All players $(\mathrm{N}=56)$ had been familiar with the LSPT protocol prior to final examination. Four soccer-specific wooden rebound planks have been placed as shown on each of the four lines marking the $12 \times 9.5 \mathrm{~m}$ grid (on the inside of planks) (Figure 2). Before placement, in the middle of each plank were attached 4 colored target areas (red, blue, green and white; $0.6 \times 0.3 \mathrm{~m}$ ). Furthermore, an aluminum stripe (sheet) of $0.1 \times 0.15 \mathrm{~m}$ was hanged in the middle of the target areas. Colored cones were used to differentiate the different zones. Participants began by the central cone with the soccer ball, and the investigator began timing the test from the moment the ball was played out of the inner rectangle. Just before the participant finished, the particular color was called out. The same examiner was used to eliminate variability among the inter-experimenters. The pass sequence was decided by 1 of 8 trial orders. Each trial consisted of 8 long passes (green and blue) and 8 short passes (white and red). Participants were informed that passes from within the passing area could only be executed. Besides, the players were informed that they would have to perform the test as quickly as possible for the best performance on the LSPT, while making the fewest mistakes. Until the last pass was completed the time was recorded. The examiner's further role was to record penalty time points accrued during the trials. So the examiner stood in such a position that it was possible to view all 4 target areas.

\subsection{Penalty Time for Following Errors Was Awarded}

Five seconds for completely missing the plank or going over the wrong target.

Three seconds to have the ball handled. 
Three seconds for missing the target area.

Two seconds from outside of the designated area for passing the ball.

Two seconds for any cone that touches the ball.

One second for each second taken over the 43 seconds allocated to complete the test.

Additionally, if the ball hit the $10-\mathrm{cm}$ aluminum stripe in the center of the target, 1 second was deducted from the total time which denotes as bonus time.

\subsection{LSPT Total Performance (TP)}

Total performance (TP) consisting of the time required to complete the test after adjusting penalties and/or bonus time.

\section{Results}

\subsection{Statistical Analyses}

All data are conveyed as mean and standard deviation (Table 1). Before using parametric tests, the condition of normality of data was verified using the Shapiro-Wilk Wtest. Statistical analysis was performed using SPSS version 18.0 (SPSS Inc., Chicago, IL, USA).

Table 1. The LSPT and Digit Ratio characteristics of the soccer players.

\begin{tabular}{lll}
\hline Test Protocol & Mean Performance & Standard Deviation $( \pm$ SD) \\
\hline Age (years) & 17.3 & 1.07 \\
Stretch stature $(\mathrm{cm})$ & 169.5 & 6.4 \\
Body mass $(\mathrm{kg})$ & 58.46 & 5.41 \\
LSPT $(\mathrm{TP})(\mathrm{sec})$ & 61.12 & 11.68 \\
Digit Ratio $(\mathrm{mm})$ & 0.957 & 0.023 \\
\hline
\end{tabular}

\subsection{Comparison Between High and Low Digit Ratio Group in Soccer Passing Accuracy}

The scores in soccer passing accuracy skill, i.e., LSPT total performance of the high digit ratio and the low digit ratio groups, were selected and categorized. To find out the significance of differences of passing skills between the high digit ratio and the low digit ratio t-test was computed separately and presented in Table 2.

Table 2. Significance of differences in mean scores in LSPT total performance between the high digit ratio and low digit ratio.

\begin{tabular}{llllllll}
\hline Measures & Digit Ratio & Mean & Variance & df & t & Sig. & Observation \\
\hline \multirow{2}{*}{ LSPT (TP) } & High & 65.57 & 111.80 & 54 & 4.87 & p $<.01$ & Significantly low digit ratio was better \\
\hline
\end{tabular}

Significance level at $54 \mathrm{df}$ at $* .05$ level $=2.01$ and at $* * .01$ level $=2.68$

\subsection{Findings}

From the Table 2, using t-tests, it was observed that the mean score in the soccer passing accuracy skill test i.e. LSPT total performance (TP) of the low digit ratio group was significantly better performer than that of the high digit ratio group.

\section{Discussion}

The present study investigates the comparative analysis between high digit ratio and low digit ratio groups under soccer passing accuracy in national youth soccer players. The key findings from this study are that the low digit ratio group is more significantly better performer than high digit ratio group. Manning and Taylor [2] investigated different types of soccer players and stated that professional, International and $1^{\text {st }}$ team players had a low digit ratio (2D:4D) than the control groups, youth teams and the players who did not represent their country, respectively. Previous researchers reported that the average digit ratio of England's international soccer players $(\mathrm{N}=37)$ is 0.94 ; the black of England's professional soccer players $(\mathrm{N}=13)$ is 0.93 ; the Brazilian soccer professional $(\mathrm{N}=99)$ is 0.93 ; the Brazilian first team (first eleven) soccer professional $(\mathrm{N}=20)$ is $0.92[26,2]$.
Presumably, the digit ratio goes down, the player's rank is higher. It is also noted that the first eleven soccer professionals from Brazil had the lowest digit ratio. In fact, Brazil has created some of the most emblematic skillful players in world soccer history [28].

The prenatal testosterone hormone calls the human mind and body an "organizational effect" before birth. The previous study reported, in men of low 2D:4D, elevated prenatal exposure to testosterone as indicated by a low digit ratio (2D:4D) refers to more aggressive/hostile behavior, especially in challenging situations [29]. Researchers Dyer et al., [30] found evidence that 2D:4D was a correlate of performance in an open skill sport in Basketball game. Researchers further stated, "organizational benefits" of prenatal testosterone may be reflect the performance of open skills of sport. The present investigators, therefore, decide to examine LSPT because the test has dynamic elements. In LSPT the players were informed that they would have to perform the test as quickly as possible while making the fewest mistakes [31]. However, the dimension of skill is where the player has mastered the ability to pick and implement the appropriate technique as defined by requirements of situation. The 
meaning of this view is that the cognitive component is a fundamental competency factor in the decision-making process. Therefore, LSPT incorporated a dynamic element so that players had to decide upon how best to control the ball, how to position themselves for the pass in relation to the targets, and so on [22].

Digit ratio is associated with a range of cognitive abilities including spatial ability [32] which may facilitate in multifaceted aspects to perform better soccer passing accuracy. Cognition plays a major role in LSPT as players must make 16 consecutive passes with two different distances. For these separate distances, the strike rate of passing force and the rate of rebound force of passing on a single trail continuously changed. Therefore players had to adapt to this changing situation. Additionally, Scharfen \& Memmert, [33] found the cognitive functions Attention Window and Working Memory are partly associated with some specific and core motor skills, whereas the sum of all cognitive, as well as all motor skills are strongly correlated each other. Zoudji et al. [34] demonstrated that Working Memory (WM) is a neural mnemonic mechanism that processes and temporally stores the information needed for complex cognitive activities and is therefore a key component of rapid decision-making. For this reason, working memory / short-term memory allows players to execute LSPT better because they are expected to memorize the particular color and position of the wooden plank. In the same way, Makrem and his team [35] studied on LSPT and argued that the oral verbalization and visualization impact of soccer technical learning for boys in schools. Moreover, it is also noted that the incorporation of cognitive elements especially decisionmaking affects the total performance of LSPT. On the other hand, Loughborough Soccer Shooting Test (LSST), which had a tactical dimension and required participants to decide how best to control the ball [22]. Researchers Islam and his team studied LSST and repeated sprint ability and found a relationship between these two variables. Researchers further argued that repeated sprint capacity is likely to affect repeated shooting performance in multifaceted aspects [36]. Similarly, LSPT incorporated repeated 16 passes within a dynamic context [22]. Therefore, present researcher, assumed that repeated motor performance with cognitive dimension can be incorporated with each other. Therefore, the digit ratio correlated with cognition may also allow motor performance better in the LSPT protocol.

Previous researchers also have admitted high prenatal testosterone hormone (low Digit Ratio) to play a significant role in the development of mammalian brains that, in neural regions with sufficient testosterone receptors, influence cell death patterns and neural connectivity [37]. Therefore, high 2D:4D (low prenatal testosterone hormone and high prenatal estrogen hormone) is one of the limitations of greater soccer passing skills in multifaceted aspects of match-play setting. The digit ratio is often believed to be a reasonable indicator of adult steroid hormones. So, men with low digit ratios report more marked testosterone surges during challenging situations [38] linked with high performance in soccer skills.

After a comparative analysis, the present study found the significance of differences between high digit ratio and low digit ratio under soccer passing accuracy was to some extent conclusive. The low digit ratio group was significantly better than that of the high digit ratio group in soccer passing accuracy test. Therefore, Hypothesis $\mathrm{H}_{1}$ : "There would be significant differences in soccer passing accuracy skills between the high digit ratio and low digit ratio soccer players" was accepted.

\section{Conclusion}

Performing soccer passing accuracy is an intelligentattacking skill with lower and higher intensity actions in large numbers. It is noted that in match-like situations, the motor action and cognitive dimension are mixes with the success of soccer passing accuracy. Amazingly, the low digit ratio may meet the demands of the soccer game's cognitive needs and motor functions as this ratio represents the masculine traits.

For all counts, and with proven findings, it is no wonder that low digit ratio has an "organizational effect" on adult male soccer players that likely to be helpful for high passing skill performance.

\section{Recommendation}

Further research can be done in the same topic with tested by adult testosterone hormone that might correspond to the more decisive results.

\section{Limitation of the Study}

In the absence of opponents during the LSPT protocol, players seem to feel mental comfort, while impersonal time pressure is the only motivation for their action. This does not seem to reflect the actual actions taken by the players in the field in a $90 \mathrm{~min}$ soccer match.

\section{Acknowledgements}

We thank Linda Wooldridge and Mathew Clement, for Figure 1 and Figure 2 for Ajmol Ali. Those two figures were obtained from other studies.

\section{Conflicts of Interest}

The authors declare that they have no competing interests.

\section{References}

[1] Bönte W, Procher VD, Urbig D, Voracek M. Digit Ratio (2D:4D) Predicts Self-Reported Measures of General Competitiveness, but Not Behavior in Economic Experiments. Front BehavNeurosci 2017; 11. https://doi.org/10.3389/fnbeh.2017.00238. 
[2] Manning JT, Taylor RP. Second to fourth digit ratio and male ability in sport: implications for sexual selection in humans. Evol Hum Behav Off J Hum Behav EvolSoc 2001; 22: 61-9.

[3] Acar H, Tutkun E. Analysis of the 2D:4D ratios of national and amateur football players. Int J ApplExercPhysiol 2019; 8: 132-7. https://doi.org/10.30472/ijaep.v8i1.326.

[4] Keshavarz M, Bayati M, Farzad B, Dakhili A, Agha-Alinejad $\mathrm{H}$. The second to fourth digit ratio in elite and non-elite Greco-Roman Wrestlers. J Hum Kinet 2017; 60: 145-51. https://doi.org/10.1515/hukin-2017-0097.

[5] Moffit DM, Swanik CB. The association between athleticism, prenatal testosterone, and finger length. J Strength Cond Res 2011; 25: 1085-8. https://doi.org/10.1519/JSC.0b013e3181d4d409.

[6] Islam MS, Kundu B. Digit ratio and soccer. Orthop Sports Med Open Access J 2019; 3 (1): 227-230. https://doi.org/10.32474/OSMOAJ.2019.03.000154.

[7] Perciavalle V, Di Corrado D, Petralia MC, Gurrisi L, Massimino S, Coco M. The second-to-fourth digit ratio correlates with aggressive behavior in professional soccer players. Mol Med Rep 2013; 7: 1733-8. https://doi.org/10.3892/mmr.2013.1426.

[8] Hsu C-C, Su B, Kan N-W, Lai S-L, Fong T-H, Chi C-P, et al. Elite collegiate tennis athletes have lower 2D: 4D ratios than those of nonathlete controls. J Strength Cond Res 2015; 29: 822-5. https://doi.org/10.1519/JSC.0000000000000681.

[9] Bull R, Benson PJ. Digit ratio (2D:4D) and the spatial representation of magnitude. Horm Behav 2006; 50: 194-9. https://doi.org/10.1016/j.yhbeh.2006.02.008.

[10] Jeevanandam S, Muthu PK. 2D:4D ratio and its implications in medicine. J ClinDiagn Res JCDR 2016; 10: 01-3. https://doi.org/10.7860/JCDR/2016/21952.9000.

[11] Golby J, Meggs J. Exploring the organizational effect of prenatal testosterone upon the sporting brain. J Sports Sci Med 2011; 10: 445-51.

[12] Mal B. Coaching Football Professional. New Delhi, India: Friends Publications; 2004.

[13] Ali A. Measuring soccer skill performance: A review. Scand J Med Sci Sports 2011; 21: 170-83. https://doi.org/10.1111/j.1600-0838.2010.01256.x.

[14] Williams AM. Perceptual skill in soccer: implications for talent identification and development. J Sports Sci 2000; 18: 737-50. https://doi.org/10.1080/02640410050120113.

[15] Russell M, Sparkes W, Northeast J, Cook CJ, Love TD, Bracken RM, et al. Changes in Acceleration and Deceleration Capacity Throughout Professional Soccer Match-Play. J Strength Cond Res 2016; 30: 2839-44. https://doi.org/10.1519/JSC.0000000000000805.

[16] De A, Ghosh S. Effect of healthy activity programmes on kinesthetic perception and self-concept among school students. Int J AdvSci Res 2016; 1: 29-33.

[17] De A, Mondal S. Improvement of Brain Function through Combined Yogic Intervention, Meditation and Pranayama: A Critical Analysis. Eur J Phys Educ Sport 2016; 13. https://doi.org/10.13187/ejpe.2016.13.89.

[18] Islam MS. Introducing drone technology to soccer coaching.
Int J Sports Sci Phys Educ 2020; 5: 1-4. https://doi.org/10.11648/j.ijsspe.20200501.11.

[19] Pruna R, Bahdur K. Cognition in Football. J Nov Physiother 2016; 6: 1-5. https://doi.org/10.4172/2165-7025.1000316.

[20] Adams D, Morgans R, Sacramento J, Morgan S, Williams M. Successful short passing frequency of defenders differentiates between top and bottom four English Premier League teams. Int J Perform Anal Sport 2013; 13. https://doi.org/10.1080/24748668.2013.11868678.

[21] BenOunis O, BenAbderrahman A, Chamari K, Ajmol A, BenBrahim M, Hammouda A, et al. Association of shortpassing ability with athletic performances in youth soccer players. Asian J Sports Med 2013; 4: 41-8. https://doi.org/10.5812/asjsm.34529.

[22] Ali A, Williams C, Hulse M, Strudwick A, Reddin J, Howarth L, et al. Reliability and validity of two tests of soccer skill. J Sports Sci 2007; 25: 1461-70. https://doi.org/10.1080/02640410601150470.

[23] Chai XJ, Jacobs LF. Digit Ratio Predicts Sense of Direction in Women. PLoS ONE 2012; 7 https://doi.org/10.1371/journal.pone.0032816.

[24] Peters M, Manning JT, Reimers S. The effects of sex, sexual orientation, and digit ratio (2D:4D) on mental rotation performance. Arch Sex Behav 2007; 36: 251-60. https://doi.org/10.1007/s10508-006-9166-8.

[25] Geschwind N, GalaburdaAM. Cerebral lateralization. Biological mechanisms, associations, and pathology: I. A hypothesis and a program for research. Arch Neurol 1985; 42: 428-59.

[26] Manning JT. Digit Ratio (A pointer to Fertility, Behavior, and Health). United States of America: Rutgers University Press; 2002.

[27] Manning JT. Resolving the role of prenatal sex steroids in the development of digit ratio. Proc Natl Acad Sci U S A 2011; 108: 16143-4. https://doi.org/10.1073/pnas.1113312108.

[28] Atkins C. Pele and the 20 Greatest Brazilian Footballers of All Time. Bleach Rep 2013. https://bleacherreport.com/articles/1485218-pele-and-the-20greatest-brazilian-footballers-of-all-time (accessed June 6, 2020).

[29] Buchholz VN, Mühle C, Factors CS on SUR, Kornhuber J, Lenz B, Gmel G, et al. Lower Digit Ratio (2D:4D) Indicative of Excess Prenatal Androgen Is Associated With Increased Sociability and Greater Social Capital. Front BehavNeurosci 2019; 13. https://doi.org/10.3389/fnbeh.2019.00246.

[30] Dyer M, Short SE, Short M, Manning JT, Tomkinson GR. Relationships between the second to fourth digit ratio (2D:4D) and game-related statistics in semi-professional female basketball players. Am J Hum Biol 2018; 30. https://doi.org/10.1002/ajhb.23070.

[31] Moal EL, Rué O, Ajmol A, Abderrahman AB, Hammami MA, Ounis OB, et al. Validation of the Loughborough Soccer Passing Test in young soccer players. J Strength Cond Res 2014: 1-9. https://doi.org/10.1519/JSC.0000000000000296.

[32] Lin H, Yeh M, Kang Y. An Investigation of the Relationship between Digit Ratio and Spatial Ability. In: Aykin N, editor. Int. Des. Glob. Dev., Berlin, Heidelberg: Springer; 2009, p. 266-73. https://doi.org/10.1007/978-3-642-02767-3_30. 
[33] Scharfen H-E, Memmert D. The Relationship Between Cognitive Functions and Sport-Specific Motor Skills in Elite Youth Soccer Players. Front Psychol 2019; 10. https://doi.org/10.3389/fpsyg.2019.00817.

[34] Zoudji B, Thon B, Debû B. Efficiency of the mnemonic system of expert soccer players under overload of the working memory in a simulated decision-making task. Psychol Sport Exerc 2010; 11: 18-26. https://doi.org/10.1016/j.psychsport.2009.05.006.

[35] Makrem Z, Sehli H, Chameseddine G, Ali HM, Abdelawi S, Maher G. Verbalization and Visualisation Effect on Football Technical Learning Using Loughborough Soccer Passing Test (LSPT). CreatEduc 2016; 7: 720-6. https://doi.org/10.4236/ce.2016.716239.
[36] Islam MS, Kundu B, Saha S. Relationship between repeated sprint ability and accuracy of soccer shooting performance in young players. Eur J Phys Educ Sport Sci 2019; 5. https://doi.org/10.5281/zenodo.3381127.

[37] Collaer ML, Hines M. Human behavioral sex differences: a role for gonadal hormones during early development? PsycholBull 1995; 118: 55-107. https://doi.org/10.1037/00332909.118.1.55.

[38] Tomkinson G, Dyer M. Finger size does matterin sports 2017. https://theconversation.com/finger-size-does-matter-in-sports82876. 